\title{
LES RÉALITÉS VIRTUELLES ET LA PRÉSENCE : DE LA CONCEPTUALISATION A L'OPÉRATIONNALISATION
}

\author{
Yasmin Shubber ${ }^{1}$
}

\section{Introduction}

Cette étude traite des facteurs influençant l'impression de réalité dans le cadre de réalités virtuelles. Les réalités virtuelles peuvent être considérées comme une nouvelle forme de simulacre, une nouvelle manière de représenter la réalité de manière sensible. Elles sont définies de diverses façons : d'une part d'un point de vue technique par le dispositif technologique qu'elles utilisent, et d'autre part d'un point de vue conceptuel par l'activation de mécanismes perceptifs, cognitifs, et l'activation de représentations. La technologie peut être, soit de type immersive, soit de type non-immersive. Précisons d'ores et déjà quelques caractéristiques essentielles de ces réalités virtuelles. Elles se présentent sous la forme d'un environnement en trois dimensions

\footnotetext{
${ }^{1}$ Assistante, Faculté de Psychologie et des Sciences de l'Education, Université de Genève.
} 
dans lequel on peut naviguer, interagir en temps réel et qui peut produire une impression de présence ${ }^{1}$.

Si les réalités virtuelles existent depuis peu de temps, elles connaissent un développement rapide. Étudier les réactions, les phénomènes qu'elles peuvent induire peut permettre de mieux les connaître et aussi de contribuer au développement de leurs applications. Leur utilisation pour étudier les liens entre l'impression de présence et l'apprentissage constitue un moyen de comprendre, et dès lors, de favoriser les conditions d'amélioration de ce dernier. Nous allons chercher à voir quels sont les facteurs pouvant déterminer l'impression de réalité, l'impression de possible ou de plausible dans les réalités virtuelles. Pour cela nous allons étudier l'impact de deux facteurs précis dans le cadre d'un jeu vidéo, une course de véhicules futuristes. Ces facteurs sont liés à deux modalités perceptives, la vision et l'audition. Le premier facteur fait varier le point de vue du joueur dans la course, soit il voit comme s'il pilotait de l'intérieur, soit il voit l'engin qu'il pilote. Le deuxième facteur fait varier le son, soit le joueur joue avec le son, soit sans. Nous allons mesurer leurs influences au moyen de l'intensité du degré de présence dans une réalité virtuelle. Cette présence est définie comme l'impression subjective d'être dans un environnement simulé2.

Cette étude est avant tout exploratoire dans la mesure où la présence (dans des réalités virtuelles) est un concept récent et encore peu étudié. Par ailleurs, l'utilisation d'un jeu vidéo considéré comme une réalité virtuelle est une innovation. Nous avons choisi ce jeu vidéo en fonction de ses aspects ludiques et motivants, ainsi que de ses qualités graphiques. Nous faisons l'hypothèse et allons vérifier que ce jeu peut donner une impression de présence et que l'intensité de cette présence variera en fonction des facteurs choisis.

1 G. Pragier et S. Faure-Pragier, "Au-delà du principe de réalité : le virtuel", Revue française de psychanalyse, $\mathrm{n}^{\circ} 1,1995, \mathrm{pp} .63-84$; J. PsoTKA, "Immersive Tutoring Systems : Virtual Reality and Education and Training, Instructional Science, $\mathrm{n}^{\circ} 23,1995$, pp. 405-431 ; P. QUÉAU, Le virtuel: vertus et vertiges, Seysell, INA/Champ Vallon, 1993.

2 W. BARFIELD and S. WEGHORST, "The Sense of Presence within Virtual Environnements : a Conceptual Framework", in Human-computer Interaction : Software and Hardware Interfaces, vol. B, Elsevier Science Publishers, 1993, pp. 699-704; M. SLATER and M. UsOH, "Presence in Immersive Virtual Environnements", in IEEE Virtual Reality International Symposium, Seattle (Wa), september 1993. 
Nous allons présenter dans un premier temps les réalités virtuelles plus en détail dans leurs aspects techniques et dans leurs aspects cognitivo-perceptifs. Ensuite nous aborderons le concept de présence dans le cadre des réalités virtuelles. Puis nous passerons à une opérationnalisation en présentant une étude faite sur la présence et les facteurs qui l'influencent dans le cadre d'une réalité virtuelle nonimmersive (un jeu vidéo). Cette étude est extraite d'un mémoire de diplôme (Diplôme d'Études Supérieures STAF à la Faculté de psychologie de 1'Université de Genève). Nous passerons en revue les hypothèses, la procédure expérimentale ainsi que les résultats de cette expérience. Pour terminer nous conclurons en discutant de manière générale de la notion de présence et des prolongements possibles de cette étude.

\section{Les réalités virtuelles : points de vue technique et cognitivo-perceptif}

Nous allons regarder plus en détail ce que sont les réalités virtuelles. Pour cela nous avons choisi de présenter un large éventail de définitions, choisies pour leur mise en évidence d'aspects techniques et cognitivo-perceptifs, et afin de montrer le flou relatif qui y est associé. Puis nous proposerons à partir de cela une définition unique sur laquelle nous baserons la suite de notre travail.

\section{Définitions basées sur la technologie}

A la fin des années 60, Sutherland inventa une technologie permettant l'immersion dans des mondes générés par ordinateur. Pour cela, il réalisa un casque équipé d'un écran d'affichage offrant une vue stéréoscopique en trois dimensions. L'affichage était relié à un dispositif de détection des mouvements de tête de l'utilisateur et permettait d'ajuster l'affichage en fonction des mouvements. De ce fait, en tournant la tête, l'utilisateur voyait comme s'il tournait vraiment la tête dans le monde généré par ordinateur. Ce dispositif est connu sous le nom de Head Mounted Display (HMD). A l'époque, la qualité des images était très pauvre. Depuis, elle n'a cessé de s'améliorer grâce au développement technologique qui permet toujours plus de puissance à un coût moindre. Ce développement s'est fait conjointement avec celui d' "input devices", c'est-à-dire de périphériques 
permettant d'introduire de l'information dans un ordinateur : il s'agit notamment de gants sensibles aux mouvements donnant des informations tactiles ("data gloves").

C'est en 1986 que Larnier a commencé à employer le terme de "réalité virtuelle" pour décrire cette technologie. Slater et Usoh introduisent cependant une nouvelle terminologie ; ils parlent d'environnement virtuel immersif. Ici le terme immersif correspond à une technologie bien précise qui s'oppose à la technologie dite nonimmersive. Selon Psotka ${ }^{1}$, une réalité virtuelle non-immersive aurait un affichage de type "desktop display", sur un écran plat (moniteur ou télévision) au lieu d'un casque, et utiliserait un clavier, une souris, etc., comme "input device" à la place du gant. Un exemple de ce type de réalité virtuelle sont les mondes créés au moyen du langage VRML (Virtual Reality Markup Language). Ces mondes, constitués d'images en trois dimensions, sont interactifs, en temps réels, navigables, et accessibles via le World Wide Web.

Aussi la différence entre une technologie immersive et nonimmersive se trouve-t-elle dans la qualité et la quantité d'informations sensorielles transmises par l'environnement technologique au participant. Avec une technologie immersive le participant perçoit l'environnement au travers d'un casque et par là a une vue stéréoscopique, alors qu'avec une technologie non-immersive le participant le perçoit à travers un écran "plat" ("destktop display") et une perception tridimensionnelle grâce à des effets de perspectives ${ }^{2}$. Une technologie immersive est plus riche au niveau de la quantité des informations sensorielles, de la qualité de leur perceptions, et du nombre de modalités perceptives possibles, qu'une technologie non-immersive.

Ceci nous montre qu'actuellement les termes de réalité virtuelle renvoient à d'autres types de technologie que celle développée à la suite des travaux de Sutherland. Il y a ainsi un élargissement du cadre théorique et de la technologie auxquels se rapporte la réalité virtuelle. La réalité virtuelle est donc à la base une terminologie se référant à une technologie bien précise (casque et gants) puis sa définition (technique) a évolué en incorporant d'autres technologies telles que celles dites non-immersives.

Mais une définition de la réalité virtuelle basée uniquement sur cet aspect technologique est incomplète. En effet, comme le souligne

1 J. PSOTKA, op. cit.

2 Ibid. 
Jonathan Steuer, ce n'est pas une raison suffisante pour occulter ses aspects conceptuels :

(...) a device-driven definition of virtual reality is unacceptable : It fails to provide any insight into the processes or effects of using these systems, fails to provide a conceptual framework from which to make regulatory decisions..., and fails to provide a method for consumers to rely on their previous experiences with other media in understanding the nature of virtual reality ${ }^{1}$.

\section{Définitions basées sur des mécanismes cognitivo-perceptifs}

Dans le paragraphe précédent, Steuer évoquait la notion de média. Nous rappellerons la définition qu'en donne Anderson :"une activité distincte qui organise la réalité en textes lisibles en vue de 1 'action"2. On peut réinterpréter la définition de la réalité virtuelle à la lumière de cette définition. Ainsi, la réalité virtuelle serait une activité intentionnelle produite au travers de messages (visuels, auditifs, etc.) construisant un univers de représentations qui serait interprété par l'utilisateur comme réalité. Dans cette définition de média, on retrouve la notion de temps introduite par Hand. Ce média n'a de sens qu'au moment de son utilisation, le système de représentation qu'il crée n'existe que pendant la durée de son utilisation.

Si l'on s'intéresse à l'expression "réalité virtuelle", précisons que le terme de "réalité" associé à celui de "virtuel" est controversé, la réalité étant par définition à l'opposé du virtuel. Les mettre ensemble est une forme de paradoxe, un oxymore. Certains chercheurs préfèrent associer à virtuel d'autres termes tels que "environnement" ou "monde". Dans ce sens Pragier et Faure-Pragier définissent le virtuel comme étant le "réalisme d'un monde à trois dimensions où le sujet agit dans 1'image"3. Ils sont proches de la

1 J. STEUER, "Defining Virtual Reality : Dimensions Determining Telepresence", Journal of Communication, vol. 42, $\mathrm{n}^{\circ} 4,1992$, pp. 73-93.

2 J.A. ANDERSON, "Examen de quelques concepts éclairant la position de l'éducateur aux médias", in Rencontre de la recherche et de l'éducation. Éducation Média, Actes du symposium de Lausanne, 27-30 juin 1988, cité par D. PERAYA, "Théories de la communication et technologies de l'information et de la communication : un apport réciproque, Actes du "Colloque mémoire et savoir à l'ère informatique", Lausanne, 3-4 nov. 1997, Université de Lausanne, (sous presse), p. 10.

3 G. Pragier et S. Faure-Pragier, op. cit., p. 68. 
définition que propose Quéau de "monde virtuel", qu'il préfère à réalité virtuelle. Selon lui :

un monde virtuel est une base de données graphiques interactives, explorable et visualisable en temps réel sous forme d'images de synthèse tridimensionnelles pouvant stimuler chacun de nos sens afin de donner le sentiment d'immersion dans l'image'.

Ces deux définitions mettent en évidence deux aspects. Le premier est celui d'action "le sujet agit dans l'image", le deuxième est celui de production "afin de donner le sentiment d'immersion"2. De plus, cette activité qu'accompagnent des images de synthèse va créer, produire à travers une perception sensorielle un sentiment d'immersion sur lequel nous reviendrons plus en détail par la suite.

Ellis, quant à lui, définit la virtualisation comme "le processus par lequel un individu interprète une impression sensorielle comme étant un objet appartenant à un environnement autre que dans celui où il existe physiquement"3. L'auteur définit trois niveaux de virtualisation : l'image virtuelle, l'espace virtuel et l'environnement virtuel. Pour les différencier, il fait référence à des technologies différentes. La différence entre ces trois types de virtualisation réside aussi dans la qualité de la perception visuelle et de l'impression de profondeur. Il y aurait d'abord l'image avec une qualité de perception relativement faible (la perception d'une image virtuelle, d'un objet en profondeur), puis l'espace avec une qualité moyenne (la perception en profondeur au moyen de textures), et enfin l'environnement de très bonne qualité (le champ de vision plus large, parallaxe du mouvement.). Pour lui un espace virtuel se définit avec des effets de perspective, des gradients de texture permettant à l'individu de percevoir un espace et des objets en trois dimensions. Ces images en perspective sont vues sur un écran (surface) plat, contrairement aux images et environnement virtuels qui eux sont perçus à travers un dispositif stéréoscopique. La virtualisation est donc pour Ellis liée à un phénomène de perception. On ne perçoit un objet que l'on va comprendre comme existant ailleurs que dans le monde physique du percevant. Il y a donc une perception sensorielle qui va être traduite, comprise comme celle d'un objet, et

1 P. QUÉAU, op. cit., p. 13.

2 G. Pragier et S. Faure-Pragier, op. cit., p. 68.

3 S.R. ElLis, "Nature and Origin of Virtual Environments : a Bibliographic Essay", Computing Systems in Engineering, vol. 2, ${ }^{\circ} 4,1991$, p. 324. 
c'est la perception qui donne à cet objet son existence : "Je perçois quelque chose, donc ce quelque chose existe, même si dans le monde physique il n'existe pas". La virtualisation apparaît donc pour cet auteur comme un processus perceptif et compréhensif. Dans l'espace virtuel c'est la qualité, la richesse, les propriétés de 1'image qui font qu'on la perçoit comme étant en trois dimensions.

Pour Hand, enfin, la réalité virtuelle se définit de la manière suivante : "nous interagissons avec quelque chose qui n'est pas réel, mais qui peut être considéré comme tel pendant qu'on l'utilise"1. Sa définition ne parle pas spécifiquement de la notion d'images, ni de perception sensorielle. Il met l'accent d'une part sur la durée d'utilisation - d'interaction - et d'autre part sur le fait d'y croire, de l'estimer : tant que je l'utilise, j'estime que c'est réel ; la réalité virtuelle n'existe qu'à travers moi, mon expérience dans le temps et parce que je lui accorde le statut d'être réel.

Pour terminer mentionnons la définition de Psotka selon laquelle des réalités virtuelles immersives peuvent créer une impression " $d$ ' $y$ être", de présence ou d'immersion. Pour lui ceci est une des principales caractéristiques des réalités virtuelles et elle les définit "the central and defining feature of VR (virtual reality), presence or immersion"2. L'impression de présence est donc un élément déterminant dans une réalité virtuelle. Cette présence serait liée à différents facteurs qui commencent à peine à être étudiés. Il y aurait des facteurs de types technologiques, sensoriels, etc. Ces facteurs seront discutés plus en détail dans la suite de cet article.

$\mathrm{Au}$ travers de ces différentes définitions, il ressort que les notions ne sont pas les mêmes pour tous. Certains auteurs parlent effectivement de réalité virtuelle, d'autres d'environnement virtuel, de monde virtuel ou encore de virtualisation. De plus chaque auteur insiste sur un point précis qui lui est propre. Ainsi, les aspects mis en évidence dans ces définitions sont : l'action, le produit, la perception-compréhension, la durée, le rôle de média (que nous avons inféré) et enfin la présence (ou immersion). Sur un plan plus concret, des notions comme monde en trois dimensions, images de synthèse interactives, explorables, visualisables, impression sensorielle, effets de perspective, temps réel, etc. interviennent au gré des définitions. A notre avis

1 C. HAND, "Other Faces of Virtual Reality", in Proceedings of the East-West International Conference on Multimedia, Hypermedia and Virtual Reality, Moscow, Sept. 14-16 1994, p. 69.

2 J. PsoTKA, op. cit., p. 14. 
ces définitions se complémentent, aucune n'est exhaustive ou complète. Chacune aborde un point important et spécifique.

Cependant, pour des exigences méthodologiques, nous allons opter pour une définition et choisir une terminologie. Nous allons donc définir la réalité virtuelle comme étant un cadre général, ayant des aspects technologiques et conceptuels, en nous basant sur ce qu'il nous a semblé le plus pertinent dans les différentes définitions mentionnées précédemment.

Nous allons d'abord définir la réalité virtuelle sous son aspect conceptuel puis sous son aspect technologique : la réalité virtuelle consiste en un environnement en trois dimensions dans lequel on peut interagir en temps réel et que l'on perçoit quasiment comme étant réel ; cet environnement peut produire une impression de présence. La réalité virtuelle est un environnement virtuel pouvant utiliser, soit une technologie dite immersive et dans ce cas l'affichage sera perçu à travers un casque (HMD), soit une technologie dite non-immersive et dans ce cas l'affichage sera perçu à travers un écran "plat" ("desktop display"). De ce fait nous désignerons par environnement virtuel immersif, une réalité virtuelle utilisant une technologie immersive, et environnement virtuel non-immersif, une réalité virtuelle utilisant une technologie non-immersive.

Psotka dans sa définition a insisté sur l'impression de présence, celle-ci serait une des conséquences de la participation à une réalité virtuelle. Ce concept de présence dans les réalités virtuelles est un point central de cette étude. Voyons maintenant ce que nous entendons par cette notion de présence, et quel est l'état de la question à ce sujet. Précisons encore que dès à présent, lorsque nous parlerons de présence, il s'agira de présence dans les réalités virtuelles; si nous voulons parler d'un autre type de présence nous le préciserons.

\section{De l'immersion à la présence}

Lorsque l'on parle de réalité virtuelle, nous avons vu qu'il est question d'immersion. Il est important de ne pas confondre ce terme avec celui de présence. En effet, "l'immersion est une technologie, une «technique d'interface entre l'homme et l'ordinateur»' et ne

1 C. CADOZ, Les réalités virtuelles, Paris, Flammarion, 1994. 
concerne pas l'état psychologique du sujet"'. L'immersion immerge un sujet dans un environnement virtuel au travers d'informations sensorielles (vue, ouie, tactilo-kinesthésies).

La présence, par contre, est "le sentiment psychologique d'être dans (being there) l'environnement dont la base technologique est l'immersion (...) n'importe quel système d'immersion n'induit pas nécessairement de présence chez tout le monde"2.

Barfield et Weghorst parlent de présence virtuelle et la définissent ainsi : "la présence virtuelle est généralement conçue comme étant un état subjectif et hypothétique de conscience et d'implication dans un environnement non-présent"3.

Steuer fait la distinction entre présence et téléprésence :

La téléprésence est définie comme étant l'expérience d'une présence dans un environnement au moyen d'un médium de communication (...) la présence se réfère aux perceptions naturelles d'un environnement, et la téléprésence se réfère à une perception médiatisée d'un environnement. (...) cet environnement peut être un environnement virtuel animé mais nonexistant synthétisé par un ordinateur (par exemple un monde animé créé dans un jeu vidéo) ${ }^{4}$.

On retrouve ici le sujet qui nous intéresse à savoir la présence dans un jeu vidéo. A travers les quelques définitions mentionnées précédemment, on peut voir que la présence (ou présence virtuelle, ou encore téléprésence) est un sentiment subjectif d'être dans un environnement virtuel : le sentiment d'y être.

On peut aussi expliquer cette "présence" par "the willing suspension of disbelief" : la suspension volontaire d'incrédulités. Hand précise que temporairement on retire tout doute au sujet de la réalité de l'expérience que l'on vit, qu'il s'agisse d'une pièce de théâtre, de la lecture d'un roman, d'une promenade dans un bâtiment virtuel. Ce faisant, l'utilisateur peut interagir comme si l'expérience était réelle. Il est intéressant de noter que suspendre toute incrédulité peut apparaître tout aussi bien dans des réalités virtuelles que dans le

1 P. VIANIN, La perception de distances virtuelles pour un observateur mobile: approche développementale, projet de thèse FAPSE, Université de Genève, 1995, p. 7.

2 M. Slater and M. Usoh, "Presence...", op. cit. cité par P. VIANIN, op. cit., p. 7.

3 W. BARFIELD and S. WEGHORST, op. cit., p. 701.

4 J. STEUER, op. cit., p. 76. C'est nous qui traduisons.

5 C. HAND, op. cit. 
spectacle d'une pièce de théâtre, d'un film au cinéma, dans la lecture d'un roman ou encore dans l'expérience d'un rêve. Ceci corrobore les dires d'auteurs tels que Barfield, Zeltzer, Sheridan et Slater ${ }^{1}$ qui soulignent que la présence peut très bien être le résultat de la lecture d'un livre ou du spectacle cinématographique, et donc n'est pas spécifique à un environnement de type virtuel. Précisons toutefois que dans notre étude, c'est la présence dans une réalité virtuelle qui nous intéresse.

Le concept de présence évoqué à propos des réalités virtuelles n'est pas nouveau. En fait il soulève un problème posé depuis longtemps déjà, notamment à propos du cinéma. Souvenons-nous du premier film des frères Lumière, 'I'Entrée d'un train en gare de La Ciotat". Ce film qui, comme son titre l'indique, montrait l'arrivée d'un train dans une gare, effraya à tel point les spectateurs qu'ils se précipitèrent vers le fond de la salle. Pour ces spectateurs, la limite entre réel et fiction, entre le monde apparaissant à l'écran et le monde réel, physique (de la salle) n'a pas été perçue. On peut donc voir que la présence et ses conséquences n'est pas un problème nouveau, mais les réalités virtuelles le réactualisent. Ceci confirme la définition de Barfield : en effet les spectateurs se sont sentis présents, étaient impliqués dans un environnement non-présent, à savoir la gare de La Ciotat.

Par ailleurs selon Hendrix ${ }^{2}$ l'objectif des créateurs de mondes virtuels serait de créer des mondes produisant une forte impression de présence chez l'utilisateur. Cependant avant d'arriver à cela il est nécessaire de connaître les facteurs affectant la présence. Nous allons les passer en revue.

\section{Les facteurs déterminant le degré de présence}

Le thème de la présence et des facteurs l'influençant est relativement récent dans le domaine des réalités virtuelles. De ce fait, il existe peu de recherches sur ce sujet. Ces facteurs sont soit technologiques et dits externes, soit psychologiques et dits internes.

1 J. PSOTKA, op. cit.

2 C. HENDRIX, Exploratory Studies on the Sense of Presence in Virtual Environments as a Function of Visual and Auditory Display Parameters, Unpublished Doctoral Dissertation, Washington, University of Washington, 1995. 
C'est à Slater et Usoh ${ }^{1}$ que nous devons la distinction entre facteurs externes et internes.

Les facteurs internes seraient la manière dont sont intériorisées les expériences d'un individu. Un exemple est donné par la manière dont des participants se sentent présents dans un environnement virtuel en fonction du mode sensoriel dominant chez eux, c'est à dire s'ils utilisent plus la vue ou l'ouïe ${ }^{2}$.

Les facteurs externes, quant à eux, correspondraient aux types de technologie utilisés pour afficher l'environnement virtuel, à des indices perceptuels, etc. Ils correspondraient donc aux types de hardware et software utilisés.

La plupart des recherches empiriques faites à ce jour ont porté sur les facteurs externes, plus précisément sur la qualité et la richesse des informations sensorielles. De plus, la technologie utilisée pour ces travaux est une technologie immersive et l'utilisateur peut aussi bénéficier d'un corps virtuel dans l'environnement. Nous allons maintenant décrire plus précisément ces différents facteurs.

\section{Facteurs internes}

Ces facteurs internes, liés au participant lui-même et à l'interindividualité, ne sont pas à négliger. Il y a par exemple la croyance, traitée par la suite, et aussi toute la subjectivité du sujet. Dans cette optique, Psotka affirme qu'il y a une grande gamme de différences individuelles dans l'expérience de l'immersion dans des environnements de réalité virtuelle. La technologie est relativement limitée et donc ce sont les différences de tempérament, de personnalité ou encore d'expériences personnelles, qui vont faire que chacun réagit différemment à ces situations limitées, ou que les positions de chacun permettent d'outrepasser les limites dans un sens ou dans l'autre. Par exemple, si des participants sont mis en présence d'images de qualité très pauvre, leurs réactions dépendront de leur imagination visuelle. En d'autres mots, l'apport technique joue un rôle et la personne complétera, l'utilisera à sa manière dans son expérience de l'environnement virtuel.

1 M. Slater and M. Usoh, "Presence...", op. cit.

2 M. SlATER and M. UsOH, "Body centred Interaction in Immersive Virtual Environments", in N. Magnenat Thalmann and D. Thalmann (eds), Artificial Life and Virtual Reality, Wiley and Sons, 1994, pp. 125-148. 
La notion de croyance est à nos yeux d'une certaine importance. En effet le participant peut avoir tendance à croire facilement par exemple à une pièce de théâtre ou à vivre dans un film au cinéma aux côtés des protagonistes, ou encore dans un roman. En cela nous rejoignons les dires de Hand" et la notion de "willing suspension of disbelief'. La personne joue le jeu d'y croire, accepte de faire comme si c'était "vrai". Il croit à ce que l'on lui propose. Le psychanalyste Mannoni précise les processus en cause dans cette suspension volontaire d'incrédulité en expliquant que

l'adhésion du spectateur au spectacle théâtral est fondée sur un processus de dénégation : c'est parce qu'il sait pertinemment que ce qu'il voit est irréel que son imaginaire est vraiment libre. Le spectateur se trouve donc dans une position clivée, dans laquelle on retrouve la dialectique de la formule " je sais bien (que ce que je vois n'est pas vrai) mais quand même (j'y crois)"2.

Le spectateur théâtral, qui peut être rapproché du spectateur de fiction, serait donc d'une part conscient de l'irréalité de ce qu'il voit, et d'autre part, se laisserait duper et participerait "d'autant plus au récit de fiction" 3 . On peut supposer qu'un participant de réalité virtuelle peut utiliser le même mécanisme de dénégation, de croyance : savoir que ce n'est pas réel, que cela n'existe pas vraiment, mais tout de même jouer le jeu d'y croire. Ainsi aura-t-il une plus grande facilité à se sentir présent dans un environnement virtuel, immersif ou non.

1 C. HAND, op. cit.

2 O. Mannoni, Clés pour l'imaginaire ou L'autre scène, Paris, Éd. du Seuil, coll. "Le Champ freudien", 1969, cité par P. FASTREZ, Analyse comparative de l'attitude documentaire et de l'attitude fictionnelle dans la réception du message iconique. Étude expérimentale, Mémoire de licence inédit en communication, Université catholique de Louvain, Département de communication, p. 41.

3 Ibid. 


\section{Facteurs externes}

Slater et Usoh ${ }^{1}$, après collaboration et discussion avec un certain nombre de chercheurs dans le domaine (Held et Durlach ${ }^{2}$, Loomis ${ }^{3}$, Sheridan $^{4}$, Zeltzer ${ }^{5}$, Heeter ${ }^{6}$, Steuer ${ }^{7}$, Barfield et Weghorst ${ }^{8}$ ), proposent les cinq facteurs suivants qui vont permettre de créer un sentiment de présence (notre traduction):

1. La richesse des informations sensorielles et la transparence.

2. La cohérence de l'environnement.

3. L'interaction entre les participants et l'environnement.

4. La ressemblance entre le "corps virtuel" du participant et le participant.

5. Le lien entre les actions du participant et leurs effets.

Dans cette étude nous allons tout particulièrement nous intéresser au premier facteur externe : la richesse des informations sensorielles et la transparence. Nous allons détailler ce facteur en nous concentrant sur les modalités perceptives que nous voulons faire varier. Pour cela nous allons présenter différents concepts théoriques sur, d'une part, la modalité auditive et, d'autre part, la modalité visuelle en abordant les images virtuelles et le point de vue.

a. La richesse de l'information sensorielle et la transparence : la modalité auditive

Hendrix $^{9}$ a fait des études sur la présence dans des environnements virtuels immersifs en faisant varier des paramètres auditifs. Elle a observé que l'impression de présence était plus forte dans une situation avec son que dans une situation sans son. Elle explique son

1 M. Slater, M. Usoh and A. STeed, "Depth of Presence in Virtual Environments", Presence, vol. 3, no 2, 1994, pp. 130-144.

2 R.M. HELD and N.I. DuRLACH, "Telepresence", Presence, vol. 1, n 1, 1992, pp. 109-112

3 J.M. LoomIs, "Distal Attribution and Presence", Presence, vol. 1, n 1, 1992, pp. 113-119.

4 T.B. SHERIDAN, "Musings on Telepresence and Virtual Presence", Presence, vol. 1, $n^{\circ} 1,1992$, pp. 120-125.

5 D. ZeLTZER, "Autonomy, Interaction and presence", Presence, vol. 1, n 1, 1992, pp. 127-132.

6 C. HeEter, "Being there: the Subjective Experience of Presence", Presence, vol. $1, n^{\circ} 2,1992$, pp. 262-269.

7 J. STEUER, op. cit.

8 W. BARFIELD and S. WEGHORST, op. cit.

9 C. HENDRIX, op. cit. 
intérêt pour le son par le fait que le système auditif est un important canal d'information dans la création d'un dispositif, d'un environnement virtuel, bien qu'il soit souvent négligé. Le son a des propriétés qui le différencie de la vision. Par exemple il peut être entendu simultanément en trois dimensions et il peut être détecté plus rapidement que des stimuli. De plus la modalité auditive serait préférée à la visuelle lorsqu'il y a saturation d'informations visuelles.

L'information auditive peut aussi compléter l'information visuelle. L'audition facilite la direction du regard et de ce fait améliore le temps de réaction à un stimulus visuel. Soulignons encore que le système auditif est sensible aux variations des signaux acoustiques à travers le temps. Selon Hendrix, dans des environnements virtuels, des signaux auditifs non-verbaux ont le potentiel de produire un médium (display) plus riche augmentant de la sorte notre sentiment de présence dans un environnement virtuel, ce qui a été le cas lorsque l'on a rajouté le son aux images animées.

Précisons qu'il n'y pas eu vraiment d'étude sur la complémentarité du son et de l'image. Seule Hendrix a comparé l'ajout du son dans un environnement virtuel à un phénomène équivalent à celui du son au cinéma. Rappelons succinctement ce qu'il en est afin de mieux comprendre le rôle que joue le son.

M. Chion dans son ouvrage sur le son au cinéma explique que :

Ce phénomène, qui asservit la localisation attribuée par le spectateur au son à la vision de sa source réelle ou supposée (ou pour les aveugles, à la situation de celle-ci dans l'espace géométral), est vérifié tous les jours des millions de fois. C'est sur lui que repose le "réalisme" du cinéma parlant et sans lui il serait impossible de croire à ces sons et à ces voix qui ne bougent pas dans la largeur de l'écran, alors que les objets et les personnages qui les émettent s'y déplacent sans arrêt ${ }^{1}$.

Le son peut donner l'impression que quelque chose est plausible mais pas forcément qu'il correspond au réel. Le son couplé à une image pas forcément réelle aura un rôle déterminant. Si l'on prend l'exemple des films fantastiques et de science-fiction notamment, "le son est mobilisé pour accréditer, par des bruitages convaincants, de nouveaux appareils, de nouvelles créatures, de nouveaux espaces"2,

1 M. ChIon, Le son au cinéma, Paris, Cahiers du cinéma, coll. "Essais", 1992, p. 29.
2 Ibid., p. 67. 
par ce procédé on va obtenir un effet de réalité. Il y a l'idée du support, de donner "du crédit" à travers une information sensorielle.

Or, c'est typiquement un des aspects de notre problématique sur les réalités virtuelles que l'on retrouve ici. Nous allons partir du principe qu'en ce qui concerne le son, les domaines du cinéma et des réalités virtuelles sont comparables.

b. La richesse de l'information sensorielle et la transparence : la modalité visuelle

On peut aborder les images virtuelles par la richesse de l'information sensorielle qu'elles offrent à leur utilisateur ou encore par le point de vue - subjectif ou objectif - qu'elles instancient.

Les images que l'on trouve dans les réalités virtuelles sont des images de synthèse ou des images virtuelles. "Une image est dite virtuelle si son origine est une description numérique dans une mémoire informatique"1.

Quelle est la particularité de ce type de représentation que sont les images de synthèse?

Les images de synthèse (ou virtuelles) sont des représentations de type iconique basées sur des relations analogiques entre ces représentations et leurs référents. Le référent est un ensemble de traits perceptifs conventionalisés, un modèle perceptif de l'objet ${ }^{2}$. Or un des intérêts de ce type de représentation réside dans le fait que ce référent peut ne pas exister. Ainsi l'image virtuelle peut renvoyer à quelque chose qui n'existe pas. Le signifiant ou représentation, entretient avec le référent des relations de transformations de l'ordre de l'analogie, de la ressemblance. Toute la force et la particularité des images des réalités virtuelles se trouvent dans cette relation analogique avec un référent qui peut renvoyer à quelque chose n'existant pas, à quelque chose n'étant pas forcément réel. Et malgré "l'irréalité" possible de ce référent, la représentation ou image virtuelle sera perçue correctement, on comprendra à quel référent elle renvoie.

Pour ce qui est du point de vue, nous allons (à nouveau) faire un parallèle entre le spectateur au cinéma et le participant d'une réalité virtuelle. Voyons ce qu'il en est du spectateur cinématographique.

1 P. LÉvy, Cyberculture. Rapport au Conseil de l'Europe, Paris, Odile Jacob, 1997, p. 87.

2 D. PERAYA, "Vers une théorie des paratextes", Recherches en communication, $\mathrm{n}^{\circ} 4$, 1995, pp. 119-156. 
Selon Jean-Pierre Meunier, lorsque le spectateur contemple des images cinématographiques, il se met à l'écart du monde des réalités externes -résistantes-, il suspend du même coup son sens des réalités extérieures et se laisse envelopper par les images.

Le caractère absolu de point de vue du spectateur sur l'irréel, lui font perdre les notions mêmes de situation et de point de vue. Le spectateur ne se sent vraiment ni près ni loin des êtres irréels qu'il perçoit, il ne se sent nulle part. (...) et il en oublie même la sensation d'avoir un point de vue ${ }^{1}$.

On peut voir que le spectateur se projette, s'identifie au personnage, tout comme le fait un joueur avec le protagoniste du jeu ou un participant d'un environnement virtuel. Cependant il y a une différence fondamentale entre le spectateur et le participant : le premier n'a aucune prise sur ce qu'il voit, il subit, il est passif, alors que le participant est actif, il interagit avec l'environnement virtuel. Mais quel est le point de vue du participant, où se sent-il ?

Le spectacle immédiatement proposé par les images demeure un "réel-pour-nous", parce qu'il est constitué par nous et que nous ne cessons d'être sujet tout-percevant ${ }^{2}$.

Pour en revenir au participant, il y a donc chez lui une certaine centration : l'environnement virtuel le ramène à lui puisque ses actions seront reproduites par le personnage à l'écran. Cependant le point de vue visuel du participant peut avoir une influence. En effet si l'on reprend le concept d'ocularisation de Gaudreault et Jost ${ }^{3}$, au cinéma, on peut observer qu'il y a un lien entre le point de vue et la centration. Le concept d'ocularisation concerne le point de vue visuel et caractérise la relation entre ce qui est vu à travers l'image et qui est censé le voir. En d'autres termes cela revient à déterminer à qui appartient le point de vue de ce que l'on voit à l'écran. Gaudreault et Jost distinguent l'ocularisation interne (primaire et secondaire) et l'ocularisation zéro.

L'ocularisation interne correspond au fait que l'on considère l'image cinématographique que l'on voit comme vue par un æil

1 J.-P. MEUnIER et D. PERAYA, Introduction aux théories de la communication, Bruxelles, De Boeck Université, coll. "Culture \& communication", 1993, p. 129.

2 Ibid., p. 137.

3 A. Gaudreault et Fr. Jost, Le récit cinématographique, Paris, Nathan, 1990. Cité par J.-P. MEUNIER et D. PERAYA, op. cit. 
correspondant à celui d'un personnage (du film) : je vois ce que ce personnage voit. L'ocularisation externe correspond au fait que l'on attribue le point de vue que l'on a sur l'image filmée à une instance externe au monde représenté. Dans ce cas c'est le statut ou la position de la caméra qui l'emporte : je ne vois pas à travers le regard d'un personnage mais à travers celui de la caméra. Pour finir, l'ocularisation zéro correspond au fait d'effacer l'existence même de la caméra.

Dans notre étude nous avons utilisé le point de vue comme deuxième facteur de notre expérience. Regardons comment on peut rapprocher les deux points de vue que nous allons utiliser et ces notions d'ocularisation. L'ocularisation interne primaire est reconnaissable au fait que l'image contient un indice qui permet d'inférer la présence d'un regard comme par exemple une déformation de l'image, la représentation d'une partie du corps au premier plan, etc. On peut lier ce principe à celui de la caméra subjective où les indices visuels nous font voir la scène comme si nous la voyions au travers des yeux du protagoniste. L'ocularisation interne secondaire est reconnaissable à la référence à un regard obtenu par les raccords, c'est celle à laquelle nous sommes le plus habitués ; on voit avec les yeux d'une tierce personne présente dans la scène.

Il semblerait que les variations du point de vue oculaire jouent un rôle important dans les phénomènes de centration et de décentration. En effet, "un récit fonctionnant à la décentration aura également tendance à donner au spectateur des indices le renvoyant à son point de vue de spectateur ou à celui de l'auteur'1. La décentration pousse à la réflexion, au recul, à une certaine distance avec ce qui est appréhendé par le regard. On peut donc voir qu'en fonction du type de point de vue qu'aura le spectateur (et le participant de réalité virtuelle), il aura une position différente, qui pourra soit être proche de la centration, soit de la décentration.

Voyons maintenant quels sont les moyens dont nous disposons pour mesurer la présence.

\section{Les mesures du degré de présence}

Peu d'études ont été menées sur la présence. Toutes ces études ont tenté de créer des mesures de la présence et ont rencontré un

1 J.-P. MEUnier et D. PERAYA, op. cit., p. 242. 
certain nombre de difficultés ${ }^{1}$. En effet la présence serait constituée de plusieurs dimensions qu'il faudrait pouvoir mesurer pour appréhender la notion de présence. La présence est définie de manière floue, les mécanismes la produisant étant mal connus. Les modèles analytiques et conceptuels la définissant sont à un niveau rudimentaire. De plus les attributions subjectives sont considérées comme problématiques dans la mesure où leur validité peut être mise en doute ${ }^{2}$. Malgré ces diverses difficultés, un certain nombre de mesure de la présence ont été établies. Ces mesures sont réparties en deux groupes : les mesures objectives et les mesures subjectives ${ }^{3}$.

Dans les mesures objectives, on trouve certaines catégories d'indicateurs : les indicateurs physiologiques (posture, tension musculaire, etc.); les indicateurs physiométriques (réponses corticales, diamètre de la pupille) ; les performances dans les tâches effectuées dans des environnements virtuels (précision, rapidité, etc.); le cadre de référence et la résolution de conflit ; le degré de désorientation (temps mis par le sujet pour ré-émerger dans la réalité).

Les mesures subjectives correspondent à une évaluation introspective de la manière dont une personne se sent présente dans un environnement virtuel. Ces mesures peuvent être faites sur le moment même, ou peuvent être des évaluations post-tests, ou encore des explications des événements ayant pu déclencher des degrés de présence plus élevés ${ }^{4}$.

Vu la nature interne et complexe de la présence, les mesures subjectives de la présence ne sont pas simples à valider. C'est pourquoi n'utiliser qu'une sorte de mesures ne paraît pas adéquat et présente peu d'intérêt. Comme le souligne Hendrix :

Presence will certainly require the use of both subjective and objectives measures where subjective evaluation of presence will represent the fundamental measure against which all objective measures will be assessed and standardized ${ }^{5}$.

Ainsi, elle propose d'utiliser de pair des mesures subjectives et objectives, les mesures subjectives étant différenciées par rapport à

\footnotetext{
C. HENDRIX, op. cit.

W. BARFIELD and S. WEGHORST, op. cit.

C. HENDRIX, op. cit.

4 Ibid.

5 Ibid., p. 7.
} 
celles objectives qui seront estimées et standardisées. C'est dans cette optique que nous avons choisi nos différents types de mesure.

Après ce tour d'horizon dans le domaine des réalités virtuelles, de la présence, des facteurs pouvant l'influencer et des moyens de la mesurer, nous allons passer à l'opérationnalisation de ces concepts en présentant une expérience.

\section{Une expérience avec un environnement virtuel non-immersif}

L'objectif de l'étude dont nous présentons un extrait a été de déterminer quels sont les facteurs pouvant influencer le degré de présence dans un jeu vidéo (une course de voitures futuristes nommée WipeOut) considéré comme étant une réalité virtuelle. Nous sommes partis de l'hypothèse que ce jeu peut être considéré, en fonction de ses aspects perceptifs et graphiques, comme un environnement virtuel non-immersif.

Nous inférons que bien que notre environnement virtuel soit nonimmersif, il est capable de produire un certain degré de présence puisque la technologie n'est pas le seul facteur responsable de la présence. Ce postulat découle du fait que les facteurs étudiés, le son et le point de vue, appartiennent à une série de facteurs dont 1'influence sur le degré de présence a été démontrée (voir la section "Les facteurs influençant le degré de présence").

Le facteur "son" a été choisi en fonction de ce que dit la littérature afin de mettre en évidence son influence. Le facteur "point de vue" a été choisi car il nous semblait intuitivement qu'il pouvait influencer l'impression de présence et nous voulions le mettre en évidence, et aussi pour faire un parallèle avec les différents points de vue au cinéma.

Rappelons que dans le jeu, le joueur peut avoir deux points de vue différents :

1. Il voit comme s'il était à l'intérieur de la navette. Nous appellerons ce point de vue "endocentré". Il correspond au concept d'ocularisation interne ${ }^{1}$.

1 A. Gaudreault et Fr. Jost, op. cit. 
2. Il voit depuis l'extérieur de sa navette et donc la voit devant lui au cours du jeu. Ce point de vue sera nommé "exocentré". Il correspond au concept d'ocularisation externe ${ }^{1}$.

D'après les connaissances acquises et les observations présentées dans la partie précédente, nous posons les hypothèses suivantes :

1. Les joueurs jouant dans la condition "point de vue endocentré" ont un degré de présence plus élevé que les joueurs jouant dans la condition "point de vue exocentré".

2. Les joueurs jouant dans la condition "avec son" ont un degré de présence plus élevé que les joueurs jouant dans la condition "sans son".

\section{Description de la procédure expérimentale}

39 sujets sont venus jouer deux fois (pré-test et test) au jeu. Leur tâche consistait à gagner la course en allant le plus vite possible. Le pré-test permettait de découvrir, de s'habituer au jeu alors que dans le test il fallait se concentrer sur le jeu et la tâche.

Les sujets étaient répartis dans quatre différentes conditions en fonction des facteurs ; les conditions étaient les suivantes :

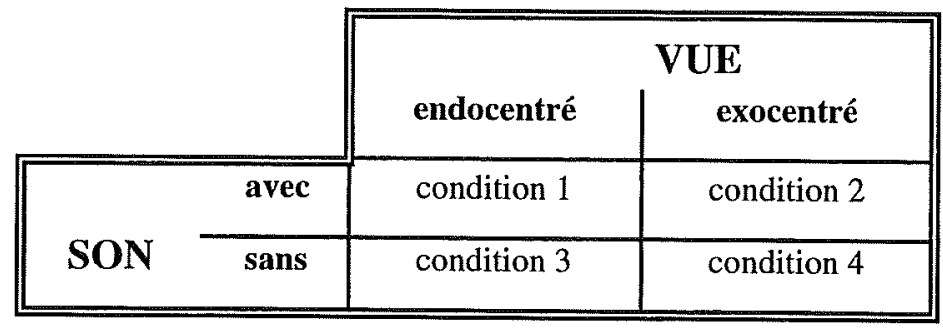

Tableau des deux facteurs et des quatre conditions

Nous avons utilisé deux types de mesures, objective et subjective.

Pour les mesures objectives : les sujets étaient filmés au cours de l'expérience et les mouvements corporels qu'ils ont effectués au cours du jeu ont été codés, puis quantifiés en différentes catégories de mouvements. Ces catégories correspondent aux parties du corps impliquées dans le mouvement; il y a 7 catégories principales : les mouvements de "Mains", de "Tête", de "Tête et mains simultané-

1 lbid. 
ment", du "Buste et des épaules", etc. Les fréquences des différents mouvements de chaque catégorie constituent les mesures objectives. Ces mesures correspondent à la catégorie des indicateurs physiologiques de Barfield et Weghorst ${ }^{1}$.

La mesure subjective a été faite au travers d'un questionnaire post-expérimental portant sur une évaluation de la présence, adapté d'un questionnaire expérimental utilisé par Slater et Usoh dans leurs travaux sur la présence ${ }^{2}$.

\section{Résultats}

Pour les résultats, nous avons fait des analyses de variance avec dans un premier temps comme facteur, le point de vue, puis dans un deuxième temps comme facteur, le son. Nos variables dépendantes sont les différentes catégories de mouvements et le degré de présence mesuré par le questionnaire.

Les résultats montrent que le point de vue a une influence sur la manière de conduire des joueurs, mais peu sur l'intensité de leur impression de présence. Il y a un effet significatif du facteur point de vue sur toutes les variables de mouvements (mesure objective) et le degré de présence mesuré par le questionnaire (mesure subjective). Ceci veut dire que l'on observe plus de mouvements et d'impression de présence dans la condition point de vue endocentré que dans celle point de vue exocentré. On retrouve cet effet significatif plus précisément pour les catégories de mouvement "Mains" et "Tête".

W. BARFIELD and S. WEGHORST, op. cit.

$2 \mathrm{M}$. SLATER and M. USOH, Post-experimental questionnaire (on the degree of presence), Unpublished ms, 1998. 
Fréquences relatives des mouvements de Mains et Tête pour le facteur vue

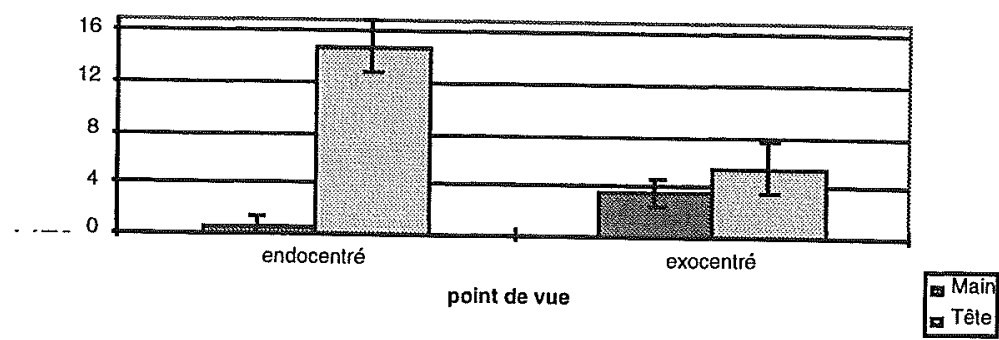

Figure 1 : Moyennes des fréquences de mouvement des catégories "Tête" et "Mains" en fonction du point de vue

Le facteur son n'a pas d'effet significatif sur l'ensemble des mesures toutes confondues (mouvements et impression de présence). Cependant des calculs portant sur chacune des variables séparément montrent des effets tendanciels pour la catégorie de mouvement "Buste-épaules" (mesure objective) et le degré de présence mesuré par le questionnaire (mesure subjective). Il y aurait plus de mouvement du buste et des épaules ainsi qu'une plus grande impression de présence lorsque les sujets jouent avec du son que sans son. $\mathrm{Ce}$ facteur semble donc avoir une plus grande influence sur l'impression de présence et il modifierait aussi la manière de conduire en la rendant plus impliquante.

Les résultats obtenus ne confirment donc pas totalement les hypothèses. Il est pourtant possible que différents comportements de conduites correspondent à différentes intensités de présence.

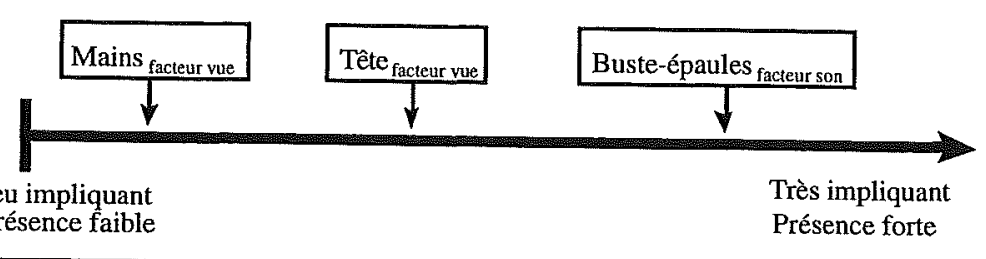

Figure 2 : Degré d'implication des trois catégories de mouvements Pour chaque catégorie est mentionné le facteur pour lequel il y a une différence significative. 
Ainsi les mouvements des "Mains" correspondraient à une conduite de téléguidage et une présence faible. En déplaçant uniquement les mains dans les virages, le joueur conduit sa navette à distance un peu comme une voiture téléguidée ; par là il se sentirait peu présent.

Pour les mouvements "Tête" dans cette conduite, la vision correspondrait à celle à travers les yeux du pilote. Le joueur bouge la tête dans les virages comme s'il voyait depuis l'intérieur de la navette. Le joueur est moyennement impliqué et présent.

Pour la catégorie "Buste-épaules", le joueur fait un mouvement de déplacement latéral du buste et des épaules dans les virages, ainsi il conduit comme s'il était dans la navette avec une accélération centripète inférée. Ce type de conduite indiquerait une grande implication et une forte présence.

\section{Conclusion}

Cette étude montre que même en utilisant une technologie dite non-immersive, un environnement virtuel peut induire une impression de présence. Les résultats montrent que le point de vue influence la manière dont les joueurs pilotent. En effet, en fonction du point de vue - endo- ou exocentré - les joueurs vont faire des mouvements dans les virages soit avec leur tête, soit avec leurs mains. Tout se passe donc comme si les joueurs voyaient depuis l'intérieur de la navette ou comme s'ils téléguidaient la navette. Mais le point de vue n'a que peu d'effet sur l'intensité de l'impression de présence. Le facteur son possède une influence plus importante. Le son semble en effet rendre la conduite plus "vécue " et provoquer un comportement plus impliqué, ce que montrent les mouvements de buste du joueur dans le virages. Si l'on se souvient de l'échelle des différents mouvements corporels, ce dernier mouvement correspond en effet à un degré d'implication plus élevé. Ce mouvement peut être compris par l'inférence d'une accélération centripète à laquelle le joueur serait soumis en se trouvant dans la navette. Le joueur rapporte d'ailleurs avoir une plus grande impression de présence dans ce cas.

Notons que les résultats observés ne peuvent sans doute être généralisés sans réserve : ils pourraient être influencés par la qualité des images et de l'animation comme par la nature ludique de 1'environnement virtuel étudié. Un jeu vidéo permet en effet d'induire une plus grande motivation chez les joueurs. 
Notre travail montre aussi la difficulté à analyser la notion de présence - voire à la mesurer - d'autant qu'à l'heure actuelle aucune définition claire n'a pu encore être établie. Cette notion présente d'ailleurs différentes dimensions qu'explorent les indicateurs que nous avons retenus et chacun d'eux mesure un aspect particulier. Les mesures de l'impression de présence vécue par les joueurs dépendent de différents facteurs interindividuels et sont subjectives; elles sont le fruit d'une introspection dont la validité dépend des sujets. Par contre les mesures objectives que nous avons relevées reflètent un comportement moins conscient des sujets. Ceux-ci sont conscients de produire des mouvements, mais ils les produisent spontanément et "automatiquement". Effectuer ces mouvements de manière préméditée et contrôlée serait très difficile et demanderait un tel effort que cela gênerait sans doute l'accomplissement de la tâche principale, à savoir gagner la course. Dès lors, les mouvements observés peuvent s'expliquer comme une conséquence de l'impression de déplacement des sujets dans le jeu, impression connue sous le nom de vection. Et les différents types de mouvements observés correspondent bien à des manières de conduire plus ou moins empathiques, et donc à un degré de présence plus ou moins élevé.

Enfin, nos résultats mettent en évidence des tendances plus que des résultats significatifs, ce qui pourrait s'expliquer, entre autres, par la variabilité intra- et inter-individuelle, par la validité de nos mesures, le dispositif expérimental lui-même ou encore par le nombre peu élevé de sujets observés. Aussi proposons-nous certains prolongements qui permettraient de confirmer les tendances observées. D'une part, notre dispositif expérimental pourrait être amélioré, qu'il s'agisse de la population, des conditions ou encore des mesures employées ; d'autre part, d'autres pistes pourraient être explorées telles que l'emploi d'autres facteurs (internes, externes, sensoriels, etc.), l'intégration d'une dimension sociale, la comparaison avec d'autres simulacres, etc.

Plus précisément, il serait important de reprendre cette étude en modifiant la composition de l'échantillon et en augmentant le nombre de sujets. On pourrait aussi, afin de réduire les effets interindividuels, faire passer différentes conditions aux mêmes sujets. Une façon d'améliorer les différentes mesures serait d'utiliser des techniques plus précises, par exemple une plate-forme de force ou un système de décodage automatique. Enfin, une comparaison entre le jeu étudié et le même type de jeu utilisant une technologie immersive (avec un 
casque HMD) apporterait certainement des informations relatives à l'importance de la technologie utilisée et à son effet sur la présence.

Quant à la dimension sociale, elle pourrait faire l'objet d'analyses particulières. On introduirait cette composante sociale par exemple en disant au joueur qu'il joue en réseau et que les autres navettes sont conduites par des personnes se trouvant dans une ou plusieurs pièces séparées. On pourrait observer la relation entre dimension sociale, motivation et présence.

Enfin, s'il est vrai que pour certains auteurs comme Psotka, l'intérêt des réalités virtuelles réside dans cette impression de présence, il faut reconnaître que ce phénomène n'est pas récent ; on l'observe dans différents simulacres inventés par l'homme au cours des siècles. Les réalités virtuelles sont simplement une nouvelle forme de simulacre utilisant une technologie relativement sophistiquée, avec des informations sensorielles (et surtout visuelles à 1'heure actuelle) très riches. Il serait intéressant de voir en quoi la présence dans ce type d'environnement se distingue et/ou se rapproche de celle que suscitent d'autres types de simulacre tel que les films, les romans, etc.

Certes nous n'avons pas répondu à toutes les questions que pose cette thématique. Nous suggérons, à partir de notre travail, quelques pistes pour les nombreuses recherches à venir. Parmi celles-ci, rappelons l'importance déterminante du son ainsi que celle de la subjectivité du joueur, sa croyance. Ces deux facteurs sont essentiels. D'abord, si le son demeure encore le plus mal connu dans le domaine, son influence est sans doute plus importante que l'on ne l'imagine. On devrait donc souhaiter qu'un plus grand nombre de recherches lui soit consacrées. Ensuite, au-delà de la richesse sensorielle des environnements virtuels et du rôle de la technologie dans ces environnements, le rôle du sujet - croyance, motivation, engagement vis-à-vis de la tâche à réaliser, etc. - ne peut être négligé. Ceci gagnerait à être entendu par tous ceux qui œuvrent dans le domaine des technologies éducatives au sens large - et qui trop souvent accordent aux aspects technologiques une prépondérance aveugle. 